Western University Scholarship@Western

Department of Economics Research Reports

Economics Working Papers Archive

1982

\title{
Search and Optimal Sample Sizes
}

Peter Morgan

Follow this and additional works at: https://ir.lib.uwo.ca/economicsresrpt

Part of the Economics Commons

Citation of this paper:

Morgan, Peter. "Search and Optimal Sample Sizes." Department of Economics Research Reports, 8208. London, ON: Department of Economics, University of Western Ontario (1982). 
ISBN : $0-7714-0345-3$

\section{RESEARCH REPORT 8208}

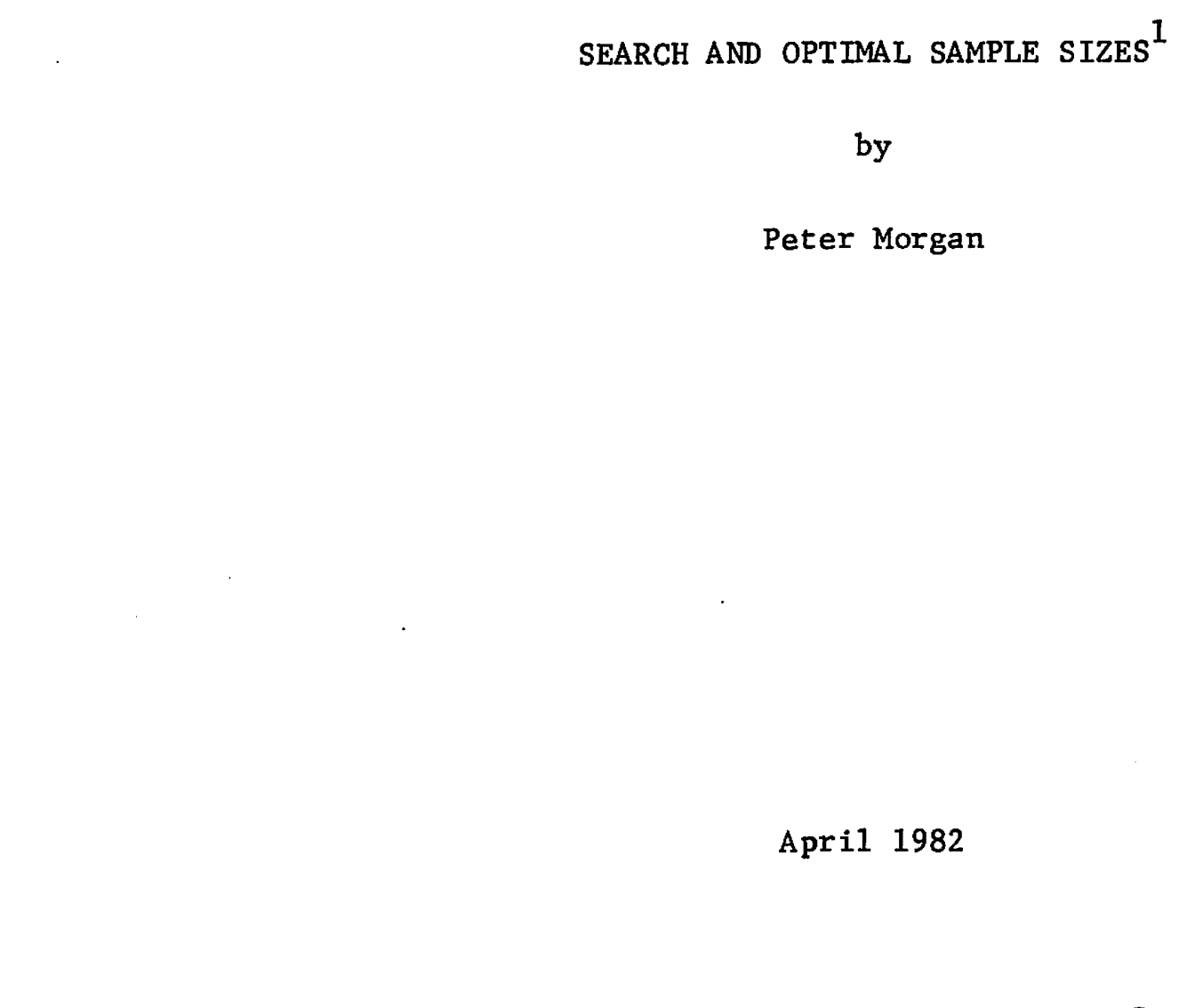

\begin{tabular}{c} 
SEARCH AND OPTIMAL SAMPLE SIZES \\
Peter Morgan \\
\hline \\
April 1982 \\
\\
\hline
\end{tabular}

\begin{tabular}{c} 
SEARCH AND OPTIMAL SAMPLE SIZES \\
Peter Morgan \\
\hline \\
April 1982 \\
\\
\hline
\end{tabular}

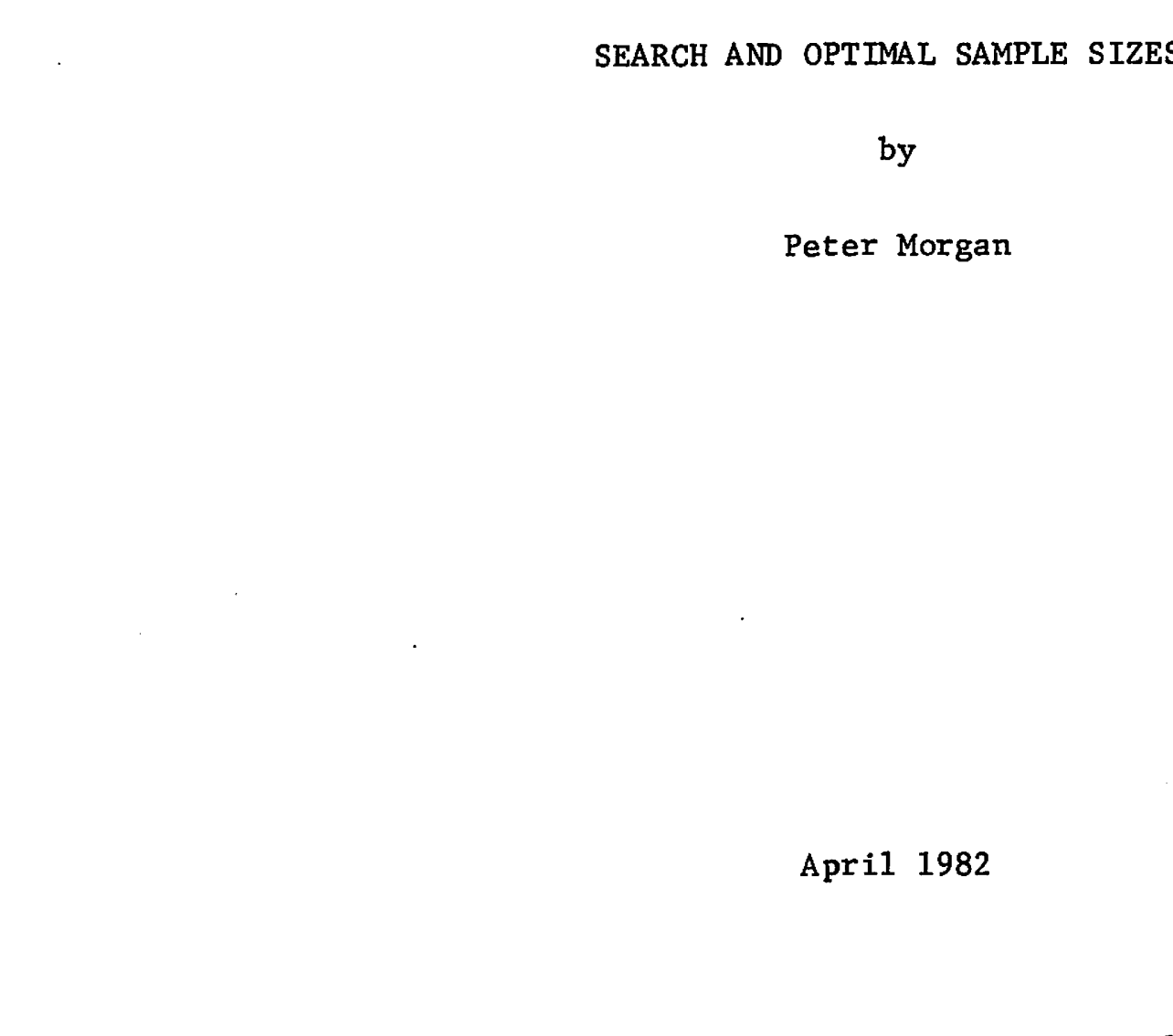

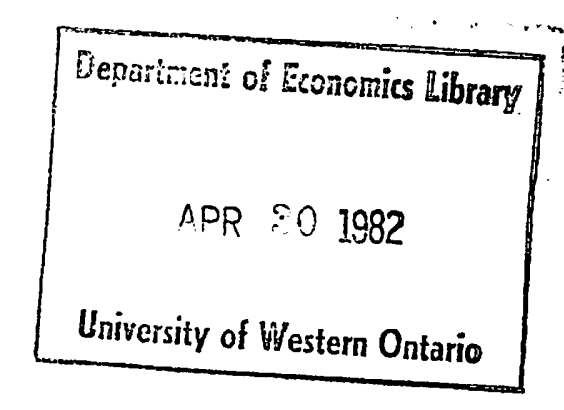




\section{INTRODUGTION}

Until very recently the optimal search literature has concerned itself with the outcomes of either sequential or fixed-sample-size (fss) search strategies only. Both of these strategies make strong presumptions about the manner in which search is conducted. Sequential strategies require that search proceeds by demanding one observation at a time (see, for example, [1, p. 267]). After a period this observation becomes available to the searcher who then chooses between stopping search or demanding a further single observation. Thus a sequential searcher's choice of sample size at each decision point is always unity until search ceases. A number of writers (e.g. [7, pp. 689-694]) have pointed out that ceteris paribus the best sequential strategy dominates the search strategy in which the length of the sequence of observations taken one at a time is fixed ex ante. This latter strategy has become known as the fixed-sample-size (fss) strategy and has been often criticized as inefficient. Recently, however, Manning and Morgan [5], [6] have shown that a fss strategy will not be dominated by a sequential strategy if the fss strategy is more realistically reinterpreted as demanding a single sample of $n$ observations simultaneously in one period.

This paper considers the class of search problems in which the searcher can choose his sample size and whether or not to stop search at each of a sequence of decision points. Sequential search problems are the set of problems in which the sample size chosen at each decision point is unity. Fss search problems are interpreted, as in [5] and [6], as the set of problems in which only one sample of observations is drawn. Clearly 
the search strategy which is optimal across the more general class of problems considered here will dominate both the best sequential and best fss search rules since they are both special cases of the optimal rule. The existence and some of the properties of this general optimal search strategy are proved in [2] and [6].

The principal purpose of this paper is to establish some of the properties of the sample size sequences which result from searching optimally with the freedom to choose both sample sizes and search duration. These properties depend upon virtually all aspects of the searcher's problems and provide necessary and sufficient conditions for the optimality of sequential search strategies within the class of problems considered here. Section 2 gives a detailed description of these problems. Section 3 extends the results of Ga1, et al. [2] for search problems with no recall. Section 4 presents results describing the effects of full recall and decision horizons upon the optimal sample size sequence. Section 5 explains the effects upon the optimal full recall and optimal no recall sample size sequences of altering the utility of not searching. Some concluding comments are presented in section 6 . 


\section{THE SEARCH PROBLEM AND THE OPTIMAL SEARCH RULE}

The search problems addressed here are a subset of those examined in [6] - Search consists of independent drawings from a set $X \subset R$, the set of reals, over which is a known p.d.f. $f(x)$. For instance, in a job search context $X$ is a set of wage rates. The searcher's objective is to maximize his expected present valued utility. He faces a sequence $\left\{t_{j}\right\}_{j=0}^{J}$ of decision points which are assumed to be equally spaced in time so that each of the $J$ periods $\left(t_{0}, t_{1}\right),\left(t_{1}, t_{2}\right), \ldots,\left(t_{J-1}, t_{j}\right)$ are of equal length. ${ }^{2}$ At any decision point $t_{j}$ the searcher must make two decisions. Firstly, he must determine $n_{j} \geq 0$, the number of observations he would draw from $X$ in the next period if he continues to search. Secondly, he must choose between continuing and stopping search at $t_{j}$. If he chooses to stop then he selects the terminal action providing the highest utility from those currently available to him。 If $n_{j}>0$ and he continues $h$ is search past $t_{j}$ then he receives observations $x_{1}^{j}, \ldots, x_{n_{j}}^{j}$ at the next decision point $t_{j+1}$. Search costs have two parts and are incurred before $t_{j+1}$ is reached. Firstly, future net gains will be discounted over the period $\left(t_{j}, t_{j+1}\right)$ by the searcher at a rate $\delta \geq 0$. Secondly, demanding $n_{j}$ observations imposes a psychic cost at $t_{j}$ of

$$
K\left(n_{j}\right) \geq 0 \text { where } K\left(n_{j}\right)=0 \text { iff } n_{j}=0 \text { and is convex. }
$$

The solution to the searcher's problem is the (optimal) search strategy which maximizes the expected present valued utility of search. Manning and Morgan [6] show this strategy has two components; an optimal stopping rule $\xi^{*}$ and an optimal sample size rule $\nu^{*}$. The role of $v^{*}$ is to determine $n_{j}^{*}$, the size of the sample which should be demanded at $t_{j}$ if search is to be continued to $t_{j+1}$. The role of $\xi^{*}$ is to decide if 
search should continue to $t_{j+1}$ or should be stopped at $t_{j} \cdot v^{*}, g^{*}$ and the structure of the searcher's problem determine the properties of the optimal sample size sequence $\left\{n_{j}^{*}\right\}_{j=0}^{J}$ Establishing these properties requires a more detailed description of the optimal search strategy and the searcher's problem.

$\Re$ denotes a terminal action set. $\Re_{j}$ is the set from which the searcher can select an observation to provide a terminal utility when he is at $t_{j}$. If the searcher has no recall then $R_{j}$ is denoted by

$$
\mathscr{R}_{j}^{\circ}=\left\{\begin{array}{l}
\left\{x_{1}^{j-1}, \ldots, x_{n_{j-1}^{j-1}}^{j-1}, \text { if } n_{j-1}>0 \text { and } j \geq 1\right. \\
\varnothing, \text { if } n_{j-1}=0 \text { or } j=0
\end{array}\right.
$$

If the searcher has complete recal1 ${ }^{3}$ then $R_{j}$ is denoted by

$$
\Re_{j}^{r}=\bigcup_{i=0}^{j} R_{i}^{o} ; j \geq 0
$$

The best terminal utility available at $t_{j}$ is

$$
\mathbf{u}_{j}^{*}=\left\{\begin{array}{l}
\bar{u}, j=0 \\
\max \left\{\bar{u}, \max _{x \in R_{j}} U(x)\right\}, 1 \leq j \leq J
\end{array}\right.
$$

where $\bar{u}$ is the utility derived from accepting none of the observations gathered. It is assumed that

$$
U(x) \text { is a continuous function of } x \text {. }
$$

Since individual observations $x$ are i.i.d. the utility levels $u=U(x)$ associated with these observations are i.i.d. with p.d.f. $g(u)=g(U(x))$ and c.d.f. $G(u)=\int_{-\infty}^{u} g(z) d z$. The p.d.f. of the maximum utility obtained from a sample of $n \geq 1$ observations is therefore

$$
\mathrm{p}(\mathrm{u} \mid \mathrm{n})=\mathrm{nG}(\mathrm{u})^{\mathrm{n}-1} \mathrm{~g}(\mathrm{u}) ; \mathrm{n} \geq 1
$$


To completely define $p(u \mid n)$ for all values of $n \geq 0$, augment $(2-6)$ by

$$
p(u \mid 0)=\left\{\begin{array}{l}
1, u=\bar{u} \\
0, u \neq \bar{u}
\end{array}\right.
$$

Let

$$
P(u \mid n)=\int_{-\infty}^{u} p(u \mid n) d u ; n \geq 0
$$

Let $w_{j}^{0}(\bar{u}, \delta, J)$ and $w_{j}^{r}\left(u_{j}^{*}, \delta, J\right)$ be the expected present valued utilities, at $t_{j}$, of continued optimal search when the searcher has no recall and complete recall respectively; $0 \leq j \leq J-1$. Similarly, let $\tilde{w}_{j}^{o}\left(\bar{u}, \delta, J, n_{j}\right)$ and $\tilde{W}_{j}^{r}\left(u_{j}^{*}, \delta, J, n_{j}\right)$ be the expected present valued utilities, at $t_{j}$, of demanding $n_{j}$ observations at $t_{j}$ and then searching optimally from $t_{j+1}$, with no recall and complete recall respectively. Search is continued at $t_{j+1}$ iff the best terminal utility at $t_{j+1}$ is smaller than the expected present value of continuing to search. ${ }^{4}$ Hence, from $(2-2),(2-6),(2-7)$ and $(2-8)$, for $0 \leq j \leq J-1$

$$
\begin{aligned}
w_{j}^{o}(\bar{u}, \delta, J) & =\max _{n_{j} \geq 0} \tilde{w}_{j}^{o}\left(\bar{u}, \delta, J, n_{j}\right) \\
& =\max _{n_{j} \geq 0}\left\{-K\left(n_{j}\right)+\frac{1}{1+\delta} E_{p}\left[\max \left\{\bar{u}, u_{,} w_{j+1}^{o}(\bar{u}, \delta, J)\right\} \mid n_{j}\right]\right\}
\end{aligned}
$$

Similarly, from $(2-3),(2-6),(2-7)$ and $(2-8)$,

$$
\begin{aligned}
w_{j}^{r}\left(u_{j}^{*}, \delta, J\right) & =\max _{n_{j} \geq 0} \tilde{w}_{j}^{r}\left(u_{j}^{*}, \delta, J, n_{j}\right) \\
& =\max _{n_{j} \geq 0}\left\{-K\left(n_{j}\right)+\frac{1}{1+\delta} E_{p}\left[\max \left\{u_{j}^{*}, u_{,} w_{j+1}^{r}\left(\max \left\{u_{j}^{*}, u\right\}, \delta, J\right)\right\} \mid n_{j}\right]\right\} .
\end{aligned}
$$

$w_{j}^{0}$ and $w_{j}^{r}$ are different functions so the sample size which maximizes (2-9) will generally differ from that which maximizes (2-10). Not unexpectedly, therefore, the properties of the optimal sample size sequence will differ between problems with no recall and problems with complete recall. Sections 
3 and 4 examine the properties of $w_{j}^{0}, w_{j}^{r}$ and the optimal sample size sequences in the absence of recall and in the presence of complete recall respectively. To avoid notational confusion the optimal sample size sequence in the absence of recall will be denoted by $\left\{n_{j}^{o J}\right\}_{j=0}^{J}$ and by $\left\{n_{j}^{r J}\right\}_{j=0}^{J}$ in the presence of complete recall.

\section{SAMPLE SIZES FOR OPTIMAL SEARCH WITHOUT RECALL}

This section considers only problems in which observations received cannot be recalled at any subsequent decision point. This results in an important simplification--the values of observations demanded now and received at the end of the current period do not affect the expected present value of optimal search continued in the next period. Gal, et al. [2, pp. 603-605] show that when $J<\infty$ the searcher's optimal sample size sequence is monotonic increasing, i.e.,

$$
n_{0}^{O J} \leq n_{1}^{0 J} \leq \ldots \leq n_{J-1}^{O J} \text { and } n_{J}^{O J} \equiv 0
$$

where each $n_{j}^{o J}$ is independent of the values of all received observations. If $\mathrm{J}=\infty$ and the searcher has no recall then the searcher's problem in determining the sample size which maximizes the expected utility of continued search is identical at each decision point. Consequently the optimal no recall sample size sequence is constant when $J=\infty$. Let $n^{0 \infty}$ denote this sample size and let

$$
W^{o}(\bar{u}, \delta, \infty)=\operatorname{limit}_{J \rightarrow \infty} w_{j}^{o}(\bar{u}, \delta, J) \text {, for any } j \geq 0 \text {. }
$$

Then, from $(2-9)$ with $J=\infty, n^{0 \infty}$ satisfies

$$
W^{o}(\bar{u}, \delta, \infty)=-K\left(n^{\infty \infty}\right)+\frac{1}{1+\delta} E_{p}\left[\max \left\{\bar{u}, u, W^{o}(\bar{u}, \delta, \infty)\right\} \mid n^{\infty \infty}\right]
$$


Proposition 1: If $\mathrm{J}=\infty$ and the searcher has no recall then

$$
\begin{aligned}
& \text { (i) } \mathrm{n}_{\mathrm{j}}^{0 \infty}=\mathrm{n}^{000} \text { for } \text { all } \mathrm{j} \geq 0 \text {, } \\
& \text { (ii) } \mathrm{n}^{000}=0 \text { iff no search is undertaken, and } \\
& \text { (iii) } \mathrm{n}^{0 \infty} \text { is the minimum of all optimal no recall sample } \\
& \text { sizes for any } 0 \leq \mathrm{j} \leq \mathrm{J}-1, \mathrm{~J} \geq 1 \text {. }
\end{aligned}
$$

Proof: (i) (3-2) is independent of $j$ so $n_{j}^{0 \infty}=n^{0 \infty}$ for all $j \geq 0$.

(ii) If $n^{0 \infty}=0$ then, from (i), $n_{j}^{\infty \infty}=0$ for all $j \geq 0$ so no search is undertaken. If no search is undertaken then $n_{0}^{0 \infty}=0$, so $n^{000}=0$ by (i).

(iii) From (i), $n_{0}^{O \infty}=n^{O \infty}$ but, from $(3-1), n_{0}^{0 J}$ is a monotonic decreasing function of $J$. Hence $n^{O \infty} \leq n_{0}^{O J} \leq n_{j}^{O J}$ for all $0 \leq \mathrm{j} \leq \mathrm{J}, \mathrm{J} \leq \infty$.

Q.E.D.

The effect of Proposition 1 is that when $J=\infty$ and the searcher has no recall, the optimal search strategy is to demand the same fixed sample size $\mathrm{n}^{0 \infty}$ at each decision point until search ceases. This is a strategy alluded to by Stigler [8, p. 218]. This strategy is analogous to a sequential search strategy since the same fss search strategy is sequentially employed at each decision point (instead of sequentially demanding a single observation at each decision point). Manning and Morgan [5] have explored the dependencies of fixed sample sizes upon various aspects of the searcher's problem and there is no reason in general for $\mathrm{n}^{\infty \infty}=1$. However, should the searcher's problem be such that $\mathrm{n}^{0 \infty}=1$ then the optimal search strategy is the best sequential strategy. 
Proposition 2: When the searcher has no recall, the optimal search rule is the best sequential search rule iff (i) $n^{0 \infty}=1$, for $J=\infty$

$$
\text { (ii) } n_{J-1}^{o J}=1 \text {, for } J<\infty \text {. }
$$

Proof: The optimal no recall search rule is a sequential rule iff

$$
n_{j}^{n j}=1 \quad \text { for a11 } 0 \leq j \leq J-1
$$

(i) If $J=\infty$, then the result follows directly from Proposition 1 and $(3-3)$.

(ii) Necessity: $\mathrm{n}_{\mathrm{J}-1}^{\mathrm{OJ}} \neq 1$ contradicts $(3-3)$.

Sufficiency: From $(3-1), n_{J-1}^{\circ J}=1$ requires

$$
0 \leq n_{0}^{O J} \leq \ldots \leq n_{J-1}^{O J}=1
$$

(3-4) is satisfied either by (a) $n_{j}^{o J}=1$ for all $0 \leq j \leq J-1$ or, for some $0<k<J-2$, by (b) $n_{j}^{o J}=0$ for $0 \leq j \leq k$ and $n_{j}^{o J}=1$ for $k+1 \leq j \leq J-1$. However, this latter sampling strategy (b) is sub-optimal since demanding no observations at $t_{0}, \ldots, t_{k}$ and then sequentially demanding observations with a decision horizon of $t_{J}$ is dominated by the sub-optimal strategy of sequentially demanding observations from $t_{0}$ constrained by a decision horizon of $t_{J-k^{*}}$ Hence $(3-4)$ must be satisfied by sampling strategy (a), i.e., $n_{j}^{\text {OJ }}=1$ for $0 \leq j \leq J-1$. 


\section{SAMPLE SIZES FOR OPTIMAL SEARCH WITH RECALL}

This section considers problems in which the searcher has complete recall. Recall means that the current best terminal utility is the current minimum for all future best terminal utilities. Discovering a higher terminal utility now raises this minimum, which raises the expected values of all future terminal utilities and so raises the expected present value of continued search. The complication introduced into the optimal sample size problem by recall is, therefore, that at any decision point the expected value of continued search depends upon the values of observations received before and at the decision point. Comparing (3-1) to Proposition 1 shows that the effect of introducing a decision horizon into a search problem with no recall is to alter the optimal sample size sequence from a constant sequence to a monotonic increasing sequence. Later in this section it is shown that introducing full recall into a search problem with no decision horizon alters the optimal sample size sequence from a constant sequence to a monotonic decreasing sequence. These opposing influences mean that in problems with both full recall and decision horizons the optimal sample size sequence will generally be neither monotonic increasing nor monotonic decreasing. Nevertheless it seems reasonable to anticipate that the influence of recall will dominate early in the search and that the influence of the decision horizon will grow as the horizon is approached. The remainder of this section presents several properties of the optimal full recall sample size sequence.

In [2, pp. 600-601] Gal, et al. show the marginal rate of return on investment in search with no recall is strictly decreasing within each 
search period, i.e., that $\tilde{W}_{j}^{o}\left(\bar{u}, \delta, J, n_{j}\right)$ is strictly concave w.r.t. $n_{j}$. The following proposition shows that $\tilde{W}_{j}^{r}\left(u_{j}^{*}, \delta, J, n_{j}\right)$ is also strictly concave w.r.t. $n_{j}$, so that the marginal rate of return on investment in search with full recall is also strictly decreasing within each search period. The proof of this proposition, and others following, assume that $\mathrm{K}(\mathrm{n})$ and $\mathrm{p}(\mathrm{u} \mid \mathrm{n})$ are both twice differentiable w.r.t. $n$. This assumption avoids much analytic complexity and does not alter the nature of the following results.

Proposition 3: The marginal expected rate of return on investment in search with full recall is strictly decreasing within each period.

Proof: From $(2-10)$, for any $1 \leq J \leq \infty$,

$$
\tilde{W}_{j}^{r}\left(u_{j}^{*}, \delta, J, n_{j}\right)=-K\left(n_{j}\right)+\frac{1}{1+\delta} \int_{-\infty}^{\infty} \max \left\{u_{j}^{*}, u_{,} w_{j+1}^{r}\left(\max \left\{u_{j}^{*}, u\right\}, \delta, J\right)\right\} p\left(u \mid n_{j}\right) d u
$$

Differentiating $(4-1)$ twice w.r.t. $n_{j}$ gives, with the use of $(2-6)$,

$$
\begin{gathered}
\frac{\partial^{2} \tilde{w}_{j}^{r}\left(u_{j}^{*}, \cdot, n_{j}\right)}{\partial n_{j}^{2}}=-\frac{\partial^{2} k\left(n_{j}\right)}{\partial n_{j}^{2}}+\frac{1}{1+\delta} \int_{-\infty}^{\infty} \max \left\{u_{j}^{*}, u_{,} w_{j+1}^{r}\left(\max \left\{u_{j}^{*}, u\right\}, \cdot\right)\right\} \\
\left(2+n_{j} \ln G(u)\right) G(u)^{n_{j}-1} g(u) \ln G(u) d u
\end{gathered}
$$

Let $z \in G^{-1}\left(e^{-2 / n} j\right)$. Then

$$
\frac{\partial^{2} p\left(u \mid n_{j}\right)}{\partial n_{j}^{2}}=\left(2+n_{j} \ln G(u)\right) G(u)^{n_{j}^{-1}} g(u) \ln G(u) \stackrel{\gtrless}{\leq} 0 \text { as } u \leqq z
$$

with $>$ for some $u<z$ and with $<$ for some $u>z_{\bullet} \max \left\{u_{j}^{*}, u_{,} w_{j+1}^{r}\left(\max \left\{u_{j}^{*}, u\right\}, \cdot\right\}\right.$ is non-decreasing in $u$ and strictly increasing in $u>\max \left\{u_{j}^{*}, y^{*}\right\}$ where $y^{*}$ is the searcher's reservation utility. Together with $(4-3)$ this gives

$$
\begin{array}{r}
\frac{\partial^{2} \tilde{w}_{j}^{r}\left(u_{j}^{*}, \cdot, n_{j}\right)}{\partial n_{j}^{2}}<-\frac{\partial^{2} k\left(n_{j}\right)}{\partial n_{j}^{2}}+\frac{1}{1+\delta} \max \left\{u_{j}^{*}, z, w_{j+1}^{r}\left(\max \left\{u_{j}^{*}, z\right\}, \cdot\right)\right\} \\
{\left[\int_{-\infty}^{z} \frac{\partial^{2} p\left(u \mid n_{j}\right)}{\partial n_{j}^{2}} d u+\int_{z}^{\infty} \frac{\partial^{2} p\left(u \mid n_{j}\right)}{\partial n_{j}^{2}} d u\right]}
\end{array}
$$




$$
\int_{-\infty}^{\infty} \frac{\partial^{2} p\left(u \mid n_{j}\right)}{\partial n_{j}^{2}} d u=\frac{\partial^{2}}{\partial n_{j}^{2}} \int_{-\infty}^{\infty} p\left(u \mid n_{j}\right) d u \equiv 0
$$

and $\frac{\partial^{2} K\left(n_{j}\right)}{\partial n_{j}^{2}} \geq 0$, by assumption (2-1). Hence $\frac{\partial^{2} \tilde{w}_{j}^{r}\left(u_{j}^{*}, \cdot n_{j}\right)}{\partial n_{j}^{2}}<0$.

Suppose a searcher samples at $t_{j}$ but is unlucky enough to receive no observation which improves his best terminal utility level $u_{j}^{*}$ so that, at $t_{j+1}, u_{j+1}^{*}=u_{j}^{*}$. Then, if $J=\infty$ the searcher faces exactly the same problem as at $t_{j}$ and so his best sample size at $t_{j+1}$ will be the same as at $t_{j}$, i.e.,

$$
u_{j}^{*}=u_{j+1}^{*} \Rightarrow n_{j}^{r \infty}=n_{j+1}^{r \infty}
$$

However, if $J<\infty$ then the searcher's problem in deciding $\mathbf{n}_{\mathbf{j}+1}^{\mathbf{r}}$ differs from deciding $n_{j}^{r J}$ because, at $t_{j+1}$, the searcher has one period less than at $t_{j}$ left to $h$ im before $h$ is decision horizon $t_{J}$ is reached. It is intuitive that, for a given best terminal utility, the expected utility of continued search will decline as the number of periods remaining to $t_{J}$ falls. What effect does this have upon the relative values of $n_{j} \mathrm{~J}$ and $\mathrm{n}_{\mathrm{j}+1}^{\mathrm{rJ}}$ ? At any decision point $t_{j}$ the optimal sample size must, as nearly as possible, equate the marginal expected gain from sampling once more and then stopping at $t_{j+1}$ with the marginal expected cost of doing so. At $t_{j}$ the expected cost of stopping at $t_{j+1}$ is the expected (foregone) value of continuing to search past $t_{j+1}$ and, if $u_{j}^{*}=u_{j+1}^{*}$, this must be at least as great as the expected cost of stopping at $t_{j+2}$. The margins w.r.t. sample size of the expected costs of stopping decline with the size of these costs so the marginal expected opportunity cost, at $t_{j}$, of 
stopping at $t_{j+1}$ is no greater than the marginal expected opportunity cost, at $t_{j+1}$, of stopping at $t_{j+2}$. However, since $u_{j}^{*}=u_{j+1}^{*}$ the marginal expected gains from sampling in one more period and then stopping are identical at $t_{j}$ and $t_{j+1}$. Consequently, the optimal sample size at $t_{j+1}$ is at least as great as at $t_{j}$ when the searcher's best terminal utility is the same at $t_{j}$ as at $t_{j+1}$.

Proposition 4: If the searcher has full recall, weakly prefers to continue to search at $t_{j+1}$ and if sampling at $t_{j}$ did not increase the searcher's best terminal utility, then the searcher's optimal sample size at $t_{j+1}$ is at. least as large as at $t_{j}$, i.e.,

$$
u_{j}^{*}=u_{j+1}^{*} \leq y^{*} \Rightarrow n_{j}^{r J} \leq n_{j+1}^{r J}
$$

Proof: (4-6) establishes the result for $J=\infty$. The remainder of the proof considers the case of $\mathrm{J}<\infty$. For any $0 \leq j \leq \mathrm{J}-1$ let $\mathrm{u}_{\mathrm{j}}^{*}=\mathrm{u}_{\mathrm{j}+1}^{*}=\mathrm{u}^{*}$ and let

$$
\begin{aligned}
\Delta & =\tilde{W}_{j}^{r}\left(u^{*}, \delta, J, n\right)-\tilde{W}_{j+1}^{r}\left(u^{*}, \delta, J, n\right) \\
& =\frac{1}{1+\delta} \int_{-\infty}^{\infty}\left[\max \left\{u^{*}, u, w_{j+1}^{r}\left(\max \left\{u^{*}, u\right\}, \delta, J\right)\right\}\right. \\
& \left.-\max \left\{u^{*}, u, w_{j+2}^{r}\left(\max \left\{u^{*}, u\right\}, \delta, J\right)\right\}\right] p(u \mid n) d u
\end{aligned}
$$

Define

$$
H(u) \equiv \max \left\{u^{*}, u, w_{j+1}^{r}\left(\max \left\{u^{*}, u\right\}, \delta, J\right)\right\}-\max \left\{u^{*}, u, w_{j+2}^{r}\left(\max \left\{u^{*}, u\right\}, \delta, J\right)\right\} \quad(4-10)
$$

so that

$$
(1+\delta) \frac{\partial \Delta}{\partial n}=\int_{-\infty}^{\infty} H(u) \frac{\partial p(u \mid n)}{\partial n} d u
$$

Since $\mathrm{y}^{*}$ is the reservation utility,

$$
\max \left\{u^{*}, u\right\} \geqq w_{k}^{r}\left(\max \left\{u^{*}, u\right\}, \delta, J\right) \text { as } \max \left\{u^{*}, u\right\} \gtreqless y^{*} \text { for } k=j+1, j+2 \text {. (4-12) . }
$$


From $(4-10),(4-11)$ and $(4-12)$ with $u^{*} \leq y^{*}$,

$$
H(u)=\left\{\begin{array}{l}
w_{j+1}^{r}\left(u^{*}, \delta, J\right)-w_{j+2}^{r}\left(u^{*}, \delta, J\right) ; u<u^{*} \\
w_{j+1}^{r}(u, \delta, J)-w_{j+2}^{r}(u, \delta, J) ; u^{*} \leq u \leq y^{*} \\
0 ; y^{*}<u
\end{array}\right.
$$

The next step in the argument is to show $\mathrm{H}(\mathrm{u})$ is monotonic decreasing.

Since $\mathrm{H}(\mathrm{u})$ is constant for $\mathrm{u}\left(\mathrm{u}^{*}, \mathrm{y}^{*}\right)$ this step reduces to showing

$$
\frac{\partial w_{j+1}^{r}(u, \delta, J)}{\partial u} \leq \frac{\partial w_{j+2}^{r}(u, \delta, J)}{\partial u} \text { for } u \in\left(u^{*}, y^{*}\right) \text {. }
$$

The general term in $w_{j+1}^{r}(u, \delta, J)$ is

$$
\begin{aligned}
w_{j+1, n}^{r}(u, \delta, J)= & -K(n)+\frac{1}{1+\delta} E_{p}\left[\operatorname { m a x } \left\{u_{,} u_{1}, \ldots, u_{n},\right.\right. \\
& \left.\left.\quad w_{j+2}^{r}\left(\max \left\{u_{,} u_{1}, \ldots, u_{n}\right\}, \delta, J\right)\right\} \mid n\right]
\end{aligned}
$$

With $u \in\left(u^{*}, y^{*}\right),(4-15)$ is

$$
\begin{aligned}
w_{j+1, n}^{r}(u, \cdot)=-K(n)+\frac{1}{1+\delta}\left[w_{j+2}^{r}(u, \cdot) G(u)^{n}\right. & +\int_{u}^{y^{*}} w_{j+2}^{r}(x, \cdot) n G(x)^{n-1} g(x) d x \\
& \left.+\int_{y^{*}}^{\infty} x n G(x)^{n-1} g(x) d x\right]
\end{aligned}
$$

Differentiating $(4-16)$ w.r.t. u gives

$$
\frac{\partial w_{j+1, n}^{r}(u, \cdot)}{\partial u}=\frac{\partial w_{j+2}^{r}(u, \cdot)}{\partial u} G(u)^{n}
$$

which establishes

$$
\frac{\partial w_{j+1, n}^{r}(u, \cdot)}{\partial u} \leq \frac{\partial w_{j+2}^{r}(u, \cdot)}{\partial u} \text { for } n \geq 0 \text { and } u \in\left(u^{*}, y^{*}\right) \text {. }
$$

Since $w_{j+1}^{r}(u, \cdot)=\max _{n \geq 0} w_{j+1, n}^{r}(u, \cdot),(4-18)$ establishes $(4-14)$. 
The final step in the proof is to use the monotonicity of $\mathrm{H}(\mathrm{u})$ to prove $(4-11)$ is negative. From $(2-6)$,

$$
\frac{\partial p(u \mid n)}{\partial n}=(1+n \cdot \ln G(u)) G(u)^{n-1} g(u)
$$

Define

$$
z^{\prime} \in G^{-1}\left(e^{-1 / n}\right)
$$

Then

$$
\frac{\partial p(u \mid n)}{\partial n} \geqq 0 \text { as } u \geqq z^{\prime}
$$

with $>$ for some $u>z^{\prime}$ and with $<$ for some $u<z^{\prime}$. Since $H(u)$ is monotonic decreasing, $(4-11)$ and $(4-21)$ together imply

$$
(1+\delta) \frac{\partial \Delta}{\partial y} \leq H\left(z^{\prime}\right) \int_{-\infty}^{z^{\prime}} \frac{\partial p(u \mid n)}{\partial n} d u+H\left(z^{\prime}\right) \int_{z^{\prime}}^{\infty} \frac{\partial p(u \mid n)}{\partial n} d u=0
$$

since $\int_{-\infty}^{\infty} \frac{\partial p(u \mid n)}{\partial n} d u \equiv 0 . \quad(4-22)$ and $(4-9)$ imply that

$$
\frac{\partial \tilde{w}_{j}^{r}\left(u^{*}, \delta, J, n\right)}{\partial n} \leq \frac{\partial \tilde{w}_{j+1}^{r}\left(u^{*}, \delta, J, n\right)}{\partial n}
$$

which implies

$$
n_{j}^{* r} \leq n_{j+1}^{* r}
$$

(4-24) and the strict concavities of $\tilde{w}_{j}^{r}(\cdot, n)$ and $\tilde{w}_{j+1}^{r}(\cdot, n) w \cdot r \cdot t \cdot n$ give the result that

$$
n_{j}^{r J} \leq n_{j+1}^{r J}
$$

The above result is illustrated in Figure 1.

The principal attraction of a sequential search strategy is that observations are viewed one at a time. This prevents the possibility of "over-investment" in information which can arise whenever two or more observations are demanded together in the one period because the best of these observations is accompanied by the remaining costly inferior observations. 
Figure 1

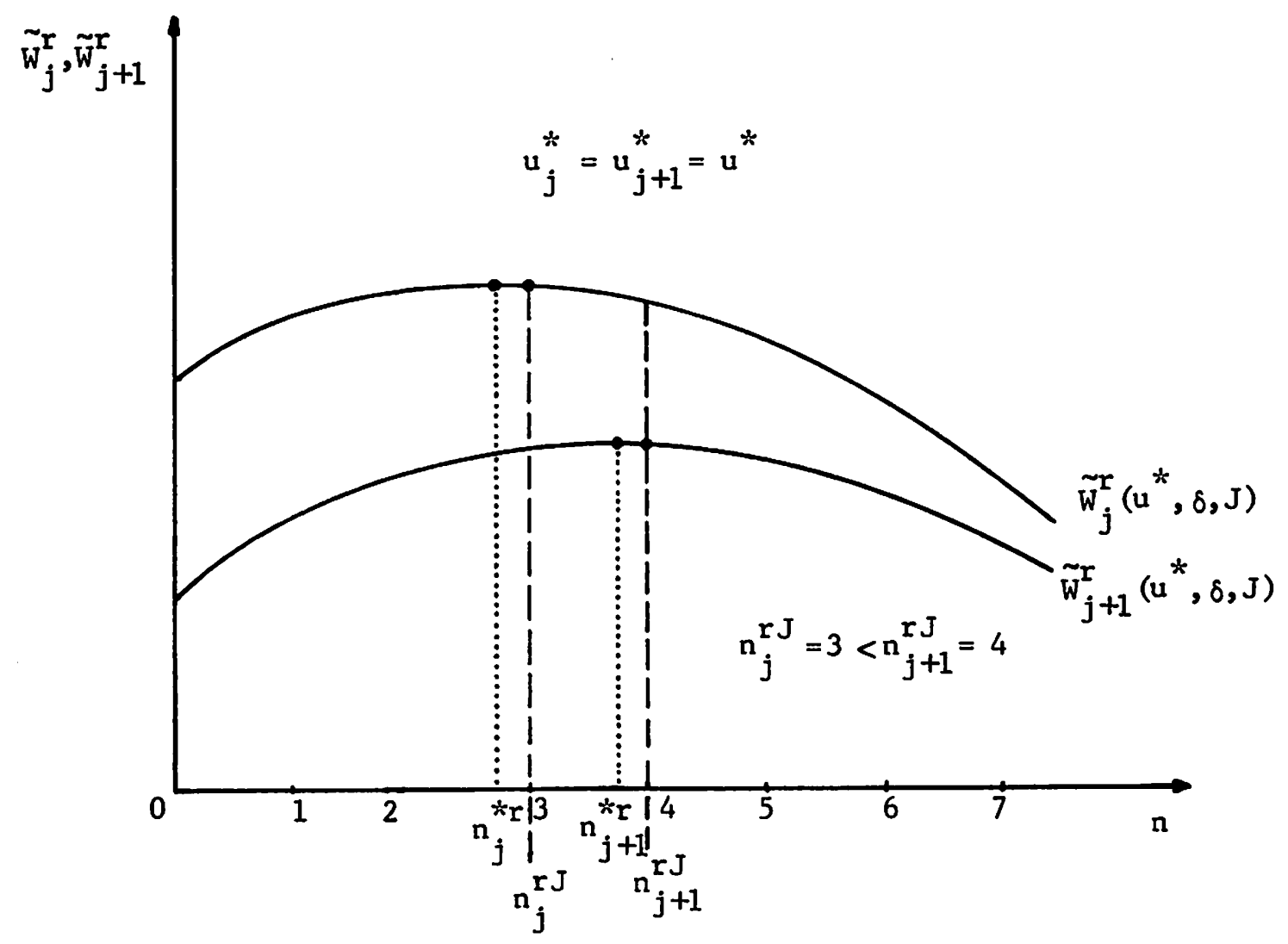


The expected cost of this "over-investment" reduces as smaller sized samples are taken within each period. On the other hand the principal effect of a finite decision horizon is to "hurry up" the searcher's information gathering since, if only a few periods are available for search, the searcher can compensate by simultaneously sampling more than one alternative in each period to amass an adequate stock of information before being forced to make a terminal decision through reaching his decision horizon. Giving the searcher extra periods (i.e., increasing $J$ ) allows the searcher to amass the same stock of information as before by demanding smaller samples within each period, thereby increasing the expected value of search by reducing the expected "over-investment" cost due to simultaneous sampling. This is the intuition behind

Corollary 1: $\mathrm{n}_{0}^{\mathrm{rJ}}$ is monotonic decreasing w.r.t. J.

Proof: At $t_{0}$ the searcher's best terminal utility is $\bar{u}$, the utility of his best non-search generated alternative. Let $J=J^{\prime}+1$. At $t_{0}$ the searcher's optimal sample size is $\mathrm{n}_{0}^{\mathrm{r}, \mathrm{J}^{\prime}+1}$. Imagine the searcher demands $\mathrm{n}_{0}^{\mathrm{r}, \mathrm{J}^{\prime}+1}$ observations at $\mathrm{t}_{\mathrm{o}}$ but receives no observation providing a utility in excess of $\vec{u}$. Then, at $t_{1}$, the searcher's problem in determining $n_{1}^{r}, J^{\prime}+1$ is exactly the same as if he had begun with only $\mathrm{J}^{\prime}$ periods and so

$$
\mathrm{n}_{1}^{\mathrm{r}, \mathrm{J}^{\prime}+1}=\mathrm{n}_{0}^{\mathrm{rJ}} \text {. }
$$

However, from Proposition 4 with $u_{0}^{*}=u_{1}^{*}=\bar{u}$,

$$
n_{0}^{r, J^{\prime}+1} \leq n_{1}^{r, J^{\prime}+1}
$$

$(4-25)$ and $(4-26)$ together show

$$
\mathrm{n}_{0}^{\mathrm{r}, \mathrm{J}^{\prime}+1} \leq \mathrm{n}_{\mathrm{o}}^{\mathrm{rJ} \mathrm{J}^{\prime}}
$$


At any decision point $t_{j}$ the optimal sample size as nearly as possible equates the marginal expected gain and the marginal expected cost from sampling again at $t_{j}$ and then stopping at $t_{j+1}$, given the current best terminal utility $u_{j}^{*}$. An increase in $u_{j}^{*}$ reduces the marginal expected gain from sampling again at $t_{j}$ and then stopping at $t_{j+1}$ since the higher is $u_{j}^{*}$, the less likely it is that a reward in excess of $u_{j}^{*}$ will be discovered at $t_{j+1}$ through sampling again at $t_{j} \cdot$ The marginal expected cost of sampling again at $t_{j}$ and then stopping at $t_{j+1}$ consists of two components. The first of these is the marginal psychic sampling cost at $t_{j}$, which is independent of $u_{j}^{*}$. The second is the marginal expected value of sampling opportunities foregone by stopping at $t_{j+1} \cdot$ This is increasing w.r.t. $u_{j}^{*}$ since $u_{j}^{*}$ can be recalled at all future decision points. Overall, therefore, the marginal expected gain and marginal expected cost of sampling again at $t_{j}$ and then stopping at $t_{j+1}$ are decreasing and increasing respectively w.r.t. $u_{j}^{*}$. A consequence of this is Proposition 5: $n_{j}^{r J}$ is a monotonic decreasing function of $u_{j}^{*}, 0 \leq j \leq J-1$. Proof: From $(2-10)$

$$
\begin{aligned}
& \begin{aligned}
\tilde{W}_{j}^{r}\left(u_{j}^{*}, \delta, J, n_{j}\right)=-K\left(n_{j}\right) & +\frac{1}{1+\delta}\left[\max \left\{u_{j}^{*}, w_{j+1}^{r}\left(u_{j}^{*}, \delta, J\right)\right\} P\left(u_{j}^{*} \mid n_{j}\right)\right.
\end{aligned} \\
& \left.\quad+\int_{*}^{\infty} \max \left\{u_{,} w_{j+1}^{r}\left(u_{,} \delta, J\right)\right\} p\left(u \mid n_{j}\right) d u\right] \\
& \frac{\partial \tilde{w}_{j}^{r}\left(u_{j}^{*}, \cdot, n_{j}\right)}{\frac{\partial u_{j}^{*}}{j}}=\frac{1}{1+\delta} \cdot \frac{\partial}{\partial u_{j}^{*}}\left[\max \left\{u_{j}^{*}, w_{j+1}^{r}\left(u_{j}^{*}, \cdot\right)\right\}\right] P\left(u_{j}^{*} \mid n_{j}\right) d u \geq 0 \\
& \frac{\partial \tilde{w}_{j}^{r}\left(u_{j}^{*}, \cdot, n_{j}\right)}{\partial u_{j}^{*} \partial n_{j}}=\frac{1}{1+\delta} \cdot \frac{\partial}{\partial u_{j}^{*}}\left[\max \left\{u_{j}^{*}, w_{j+1}^{r}\left(u_{j}^{*}, \cdot\right)\right\}\right] \frac{\partial P\left(u_{j}^{*} \mid n_{j}\right)}{\partial n_{j}} \leq 0
\end{aligned}
$$


since $P\left(u_{j}^{*} \mid n_{j}\right)=G\left(u_{j}^{*}\right)^{n}$ is decreasing w.r.t. $n_{j} \cdot$

(4-30) and the optimality condition $\frac{\partial \tilde{w}_{j}^{r}\left(u_{j}^{*}, n_{j}^{*}\right)}{\partial n_{j}} \leq 0$ imply that $n_{j}^{* r}$ is non-increasing w.r.t. $u_{j}^{*}$ and, from the concavity of $\tilde{W}_{j}^{x}\left(u_{j}^{*}, \cdot, n_{j}\right)$ w.r.t. $n_{j}$, that $\mathrm{n}_{j}^{\mathrm{rJ}}$ is also non-increasing w.r.t. $\mathbf{u}_{j}^{*}$.

This result is illustrated below in Figure 2.

The problem of describing the optimal sample size sequence with recall is greatly simplified if $J=\infty$ since then the searcher does not face a decision horizon. This simplification results from the fact that when $J=\infty$ the number of search periods remaining to the searcher does not decrease as his search proceeds. For this reason the expected value of search continued past any decision point $t_{j}$ is independent of $j$ and increasing in the best available terminal utility $\mathbf{u}_{j}^{*} \cdot(4-6)$ explained that if sampling at $t_{j}$ did not improve the searcher's best terminal utility (i.e., if $u_{j}^{*}=u_{j+1}^{*}$ ) then the searcher's problem at $t_{j+1}$ is exactly the same as at $t_{j}$ and, accordingly, $n_{j}^{r \infty}=n_{j+1}^{r \infty}$. Suppose instead that sampling at $t_{j}$ does improve the searcher's best terminal utility, i.e., $u_{j+1}^{*}>u_{j}^{*}$ Then since $u_{j+1}^{*}$ is always recoverable at all decision points past $t_{j+2}$, the opportunity cost of stopping search is higher at $t_{j+2}$ than at $t_{j+1} \cdot$ The marginal expected cost of stopping search is therefore higher at $t_{j+2}$ than at $t_{j+1}$ - Conversely, the expected increase in the best terminal utility level from drawing $n$ observations and stopping at the next decision point falls as the current best terminal utility level increases and is therefore smaller at $t_{j+1}$ than at $t_{j}$. Reduced marginal expected gains and increased marginal expected costs from sampling together reduce the optimal sample size. 
Figure 2

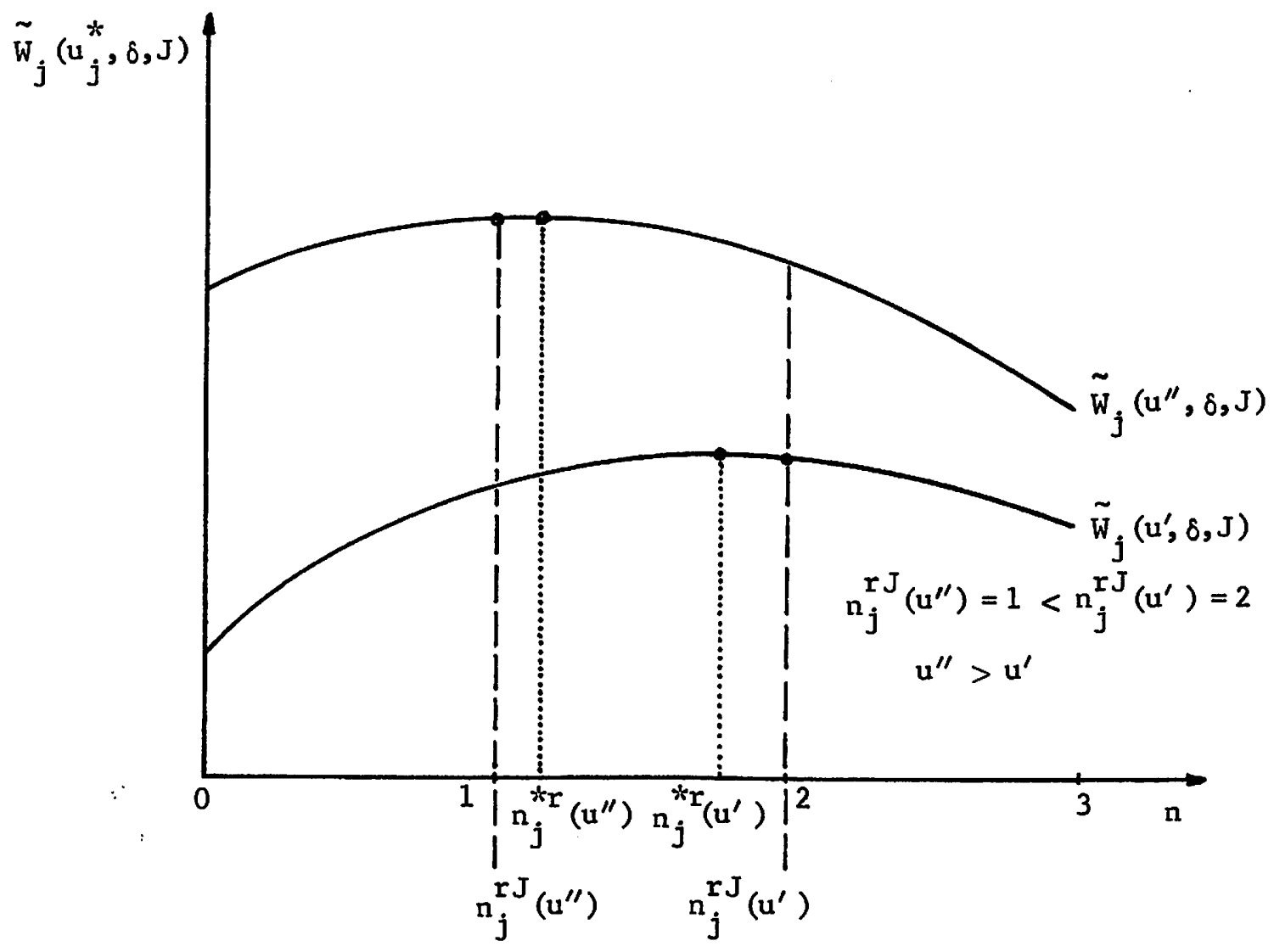


Proposition 6: If $\mathrm{J}=\infty$ and the searcher has complete recal1, then

and

(i) $n_{j}^{r \infty}=n_{j+1}^{r \infty}$ if $u_{j}^{*}=u_{j+1}^{*}$,

(ii) $n_{j}^{r \infty} \geq n_{j+1}^{r \infty}$ if $u_{j}^{*}<u_{j+1}^{*}$

Proof: (i) is established by $(4-6)$.

(ii) if $\mathrm{J}=\infty$ then

$$
\tilde{W}_{j}^{r}(u, \delta, \infty, n) \equiv \tilde{W}_{j+1}^{r}(u, \delta, \infty, n)
$$

Hence, from $(4-30)$ with $u_{j}^{*}<u_{j+1}^{*}$,

$$
\frac{\partial \tilde{w}_{j}^{r}\left(u_{j}^{*}, \delta, \infty, n_{j}\right)}{\partial n_{j}}>\frac{\partial \tilde{w}_{j+1}^{r}\left(u_{j+1}^{*}, \delta, \infty, n_{j+1}\right)}{\partial n_{j+1}}
$$

(4-32) and the optimality condition $\frac{\partial \tilde{W}^{r}\left(\cdot, n^{*}\right)}{\partial n} \leq 0$ imply $n_{j}^{*_{r}} \geq n_{j+1}^{*_{r}}$ which, with the concavity of $\tilde{w}_{j}^{r}$ and $\tilde{w}_{j+1}^{r}$, implies $n_{j}^{r \infty} \geq n_{j+1}^{r \infty}$.

The search literature has paid considerable attention to search problems in which $J=\infty$ and the searcher has recall. Many of those articles have imposed the restriction that search must be conducted sequentially. This leads, in general, to a search strategy which is suboptimal in the class of search problems considered here since there is no reason for $n_{0}^{r \infty}=1$ in general and this is a necessary and sufficient condition for the optimal search strategy to be sequential.

Corollary 2: If $\mathrm{J}=\infty$ and the searcher has complete recall, then the best sequential search rule is optimal iff $\mathrm{n}_{0}^{\mathrm{r \infty}}=1$.

Proof: Necessity: If optimal search is sequential then $n_{0}^{r \infty}=1$. Sufficiency: If $\mathrm{n}_{0}^{\mathrm{r} \infty}=1$ then, by Proposition 6,

$$
1=n_{0}^{r \infty} \geq n_{1}^{r \infty} \geq \cdots \geq n_{j}^{r \infty} \geq \cdots \geq 0
$$


i.e., optimal search consists of drawing one observation sequentially until search ceases.

Furthermore, it appears that the sets of conditions under which $\mathrm{n}_{0}^{\mathrm{r \infty}}=1$ are not particularly general, indicating that sequential search strategies may be optimal for a relatively small subset of the problems considered here. One such subset of problems is identified in [6, pp. 16-21].5 These considerations do not, however, remove the possibility that the subset of problems for which sequential search is optimal contains those search problems frequently encountered in reality.

One further implication of Proposition 5 allows a comparison of the last elements of the optimal full recall and optimal no recall sample size sequences. If just one search period remains then the ability to recall offers revealed by sampling at $t_{J-1}$ is of no advantage since search must cease at $t_{J}$, the same instant that offers demanded at $t_{J-1}$ are revealed to the searcher. At $\mathrm{t}_{\mathrm{J}-1}$, therefore, the problems of determining the full recall and no recall optimal sample sizes are identical except that a searcher with recall may be able to recall a terminal utility $u_{J-1}^{*}>\bar{u}$, the only terminal utility which can be "recalled" by a searcher unable to recall previous offers. In each case the searcher's cost function at $t_{\mathrm{J}-1}$ reduces to just $\mathrm{k}\left(\mathrm{n}_{\mathrm{J}-1}\right)$, the psychic sampling cost function. Thus if $u_{J-1}^{*}=\bar{u}$ then $n_{J-1}^{r J}=n_{J-1}^{o J}$. However, if $u_{J-1}^{*}>\bar{u}$ then the marginal expected gain to the searcher with recall from sampling again at $t_{\mathrm{J}-1}$ is smaller than for the searcher with no recall and, consequently, $n_{J-1}^{r J} \leq n_{J-1}^{0 J}$. Proposition 7: If $\mathrm{J}<\infty$ then, at $\mathrm{t}_{\mathrm{J}-1}$, the optimal full recall sample size is no greater than the optimal no recall sample size. 


\section{Proof: At $t_{\mathrm{J}-1}$,}

$$
\begin{aligned}
& \tilde{\mathrm{w}}_{\mathrm{J}-1}^{\mathrm{O}}\left(\overline{\mathrm{u}}, \delta, \mathrm{J}, \mathrm{n}_{\mathrm{J}-1}\right) \equiv \tilde{\mathrm{W}}_{\mathrm{J}-1}^{\mathrm{r}}\left(\overline{\mathrm{u}}, \delta, \mathrm{J}, \mathrm{n}_{\mathrm{J}-1}\right)=\max _{\mathrm{J}-1}\left\{-\mathrm{K}\left(\mathrm{n}_{\mathrm{J}-1}\right)+\frac{1}{1+\delta} \mathrm{E}_{\mathrm{p}}\left[\max \{\overline{\mathrm{u}}, \mathrm{u}\} \mid \mathrm{n}_{\mathrm{J}-1}\right]\right\} \\
& \text { so } \quad \mathrm{n}_{\mathrm{J}-1}^{\mathrm{OJ}} \equiv \mathrm{n}_{\mathrm{J}-1}^{\mathrm{rJ}}(\overline{\mathrm{u}}) . \quad \text { By }(2-4), \mathrm{u}_{\mathrm{J}-1}^{*} \geq \overrightarrow{\mathrm{u}} \text { so, by Proposition } 8, \\
& \mathrm{n}_{\mathrm{J}-1}^{\mathrm{OJ}} \equiv \mathrm{n}_{\mathrm{J}-1}^{\mathrm{rJ}}(\overline{\mathrm{u}}) \geq \mathrm{n}_{\mathrm{J}-1}^{\mathrm{rJ}}\left(\mathrm{u}_{\mathrm{J}-1}^{*}\right) .
\end{aligned}
$$

\section{THE FALLBACK UTILITY}

$\bar{u}$ is the highest utility available if no search is undertaken. If a sample of size $n$ is drawn from a population with p.d.f. $g(u)$ then

$$
E\left[\max \left\{\bar{u}_{,} u_{1}, \ldots, u_{n}\right\}\right]=\bar{u}_{G}(\bar{u})^{n}+\int_{\bar{u}}^{\infty} u n G(u)^{n} g(u) d u
$$

If $\bar{u}$ is lower than any of the utilities available from search (i.e., if $G(\bar{u})=0$ ) then a decrease in $\overline{\mathrm{u}}$ will not affect expectations such as $(5-1)$ and will therefore affect neither $w_{j}^{0}(\bar{u}, \delta, J)$ nor $w_{j}^{r}\left(u_{j}^{*}, \delta, J\right)$ for any $j=0, \ldots, J$. Hence if $G(\bar{u})=0$ then a decrease in $\bar{u}$ will not affect any optimal sample sizes. However, if $G(\bar{u})>0$ then a change in $\bar{u}$ affects the gains from search and the optimal sample sizes. For instance, an increase in $\bar{u}$ reduces the gains to be made from searching and this results in a decrease in the intensity with which search is undertaken.

Proposition 8: $n_{j}^{o J}$ and $n_{j}^{r J}$ are both monotonic decreasing functions of $\bar{u}$, for $j=0, \ldots, J-1 ; J \leq \infty$.

Proof: (i) Full Recall: $(2-4)$ shows $u_{j}^{*}$ is a monotonic increasing function of $\overline{\mathrm{u}}$ so, by Proposition $5, \mathrm{n}_{\mathrm{j}}^{\mathrm{rJ}}$ is a monotonic decreasing function of $\overline{\mathrm{u}}$.

(ii) No Recall: Consider two values of $\overline{\mathrm{u}} ; \overline{\mathrm{u}}^{\prime \prime}>\overline{\mathrm{u}}^{\prime}$. Let 


$$
\begin{aligned}
& (1+\delta) \Delta=(1+\delta)\left(\tilde{w}_{j}^{0}\left(\bar{u}^{\prime \prime}, \delta, J, n_{j}\right)-\tilde{w}_{j}^{0}\left(\bar{u}^{\prime}, \delta, J, n_{j}\right)\right) \\
& =\int_{-\infty}^{\infty}\left[\max \left\{\bar{u}^{\prime \prime}, u, w_{j+1}^{0}\left(\bar{u}^{\prime \prime}, \delta, J\right)\right\}-\max \left\{\bar{u}^{\prime}, u_{,} w_{j+1}^{0}\left(\bar{u}_{,}^{\prime}, \delta, J\right)\right\}\right] p\left(u \mid n_{j}\right) d u \\
& \max \left\{\bar{u}^{\prime}, w_{j+1}^{0}\left(\overline{u^{\prime}}, \delta, J\right)\right\} \\
& =\int_{-\infty}\left[\max \left\{\bar{u}^{\prime \prime}, w_{j+1}^{o}\left(\bar{u}^{\prime \prime}, \delta, J\right)\right\}-\max \left\{\bar{u}^{\prime}, w_{j+1}^{o}\left(\bar{u}^{\prime}, \delta, J\right)\right\}\right] p\left(u \mid n_{j}\right) d u \\
& \max \left\{\vec{u}^{\prime \prime}, \mathrm{w}_{\mathrm{j}+1}^{0}\left(\overline{\mathrm{u}}^{\prime \prime}, \delta, J\right)\right\} \\
& +\int\left[\max \left\{\bar{u}^{\prime \prime}, w_{j+1}^{0}\left(\vec{u}^{\prime \prime}, \delta, J\right)\right\}-u\right] p\left(u \mid n_{j}\right) d u \\
& \max \left\{\bar{u}^{\prime}, w_{j+1}^{o}\left(\bar{u}^{\prime}, \delta, J\right)\right\}
\end{aligned}
$$

Define

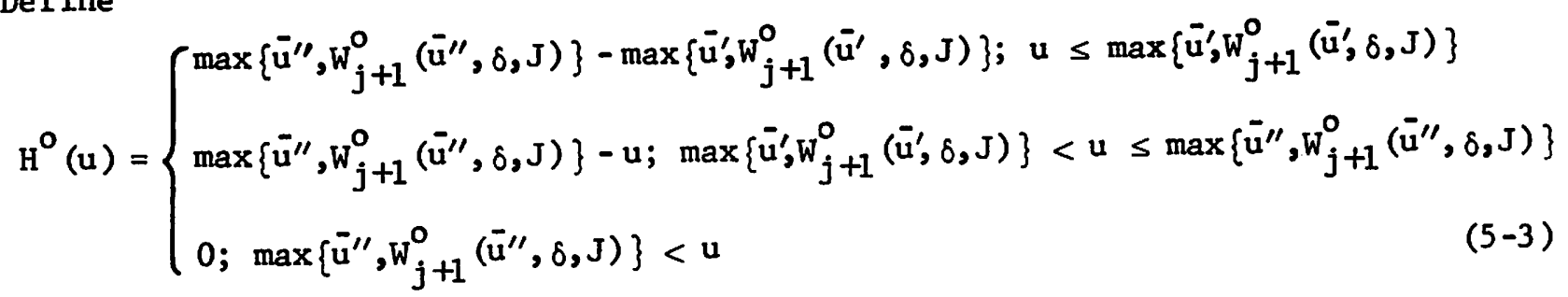

$\mathrm{H}^{\circ}(\mathrm{u})$ is monotonic decreasing w.r.t. $u$, so

$$
\begin{aligned}
(1+\delta) \frac{\partial \Delta}{\partial n_{j}} & =\int_{-\infty}^{\infty} H^{o}(u) \frac{\partial p\left(u \mid n_{j}\right)}{\partial n_{j}} d u \\
& <H^{o}\left(z^{\prime}\right)\left[\int_{-\infty}^{z^{\prime}} \frac{\partial p\left(u \mid n_{j}\right)}{\partial n_{j}} d u+\int_{z^{\prime}}^{\infty} \frac{\partial p\left(u \mid n_{j}\right)}{\partial n_{j}} d u\right]=0,
\end{aligned}
$$

where $z^{\prime}$ is defined by $(4-20)$. Hence

$$
\frac{\partial \tilde{w}_{j}^{o}\left(\bar{u}^{\prime \prime}, \delta, J, n_{j}\right)}{\partial n_{j}}<\frac{\partial \tilde{w}_{j}^{o}\left(\bar{u}^{\prime}, \delta, J, n_{j}\right)}{\partial n_{j}}
$$

which, since $\tilde{W}_{j}^{o}\left(\cdot, n_{j}\right)$ is strictly concave w.r.t. $n_{j}$, implies

$$
n_{j}^{O J}\left(\bar{u}^{\prime \prime}\right) \leq n_{j}^{O J}(\bar{u}) \text {. }
$$




\section{CONCLUDING REMARKS}

This paper presents a collection of results concerning the sizes of samples drawn when the searcher can choose both the duration of his search and his sample sizes at each of a sequence of decision points. Sequential and fixed-sample-size strategies are special cases of the optimal search strategy. The effect of recall in these problems is, in essence, to generate a monotonic decreasing sequence of optimal sample sizes, while a fixed decision horizon generates a monotonic increasing sample size sequence. Problems containing both recall and fixed decision horizons produce optimal sample size sequences with properties which are mixtures of these opposing influences. The results on optimal sample sizes provide necessary and sufficient conditions for the optimality of sequential search strategies.

The results are obtained subject to assumptions which exclude some phenomena considered elsewhere in the search literature. These exclusions include learning and the possibilities of different reward distributions and different cost functions in each period. Even so, the results still predict search behavior commonly observed in reality. One major exclusion is that of financial search costs. Manning and Morgan [5] have shown that the manner in which financial search costs affect an optimal sample size depends upon the structure of the search problem being examined. For example, if the searcher's preferences imply a marginal utility of expenditure which is a decreasing function of wealth then, ceteris paribus, the marginal utility cost of additional observations will rise as search proceeds and the searcher's net wealth declines. However, this information alone does not guarantee that the marginal disutility of psychic and 
financial search costs combined is increasing in the number of observations taken. Search for prices reveals different best prices which, in turn, affect marginal utilities. Without detailed information concerning how this occurs, it is not possible to establish the properties of the marginal overall cost of search function. Many cases may arise. 


\section{Footnotes}

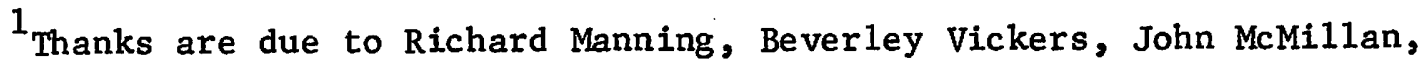
Jim Markusen, Glenn MacDonald and the Western Microeconomic Theory Workshop group for helpful comments. Responsibility for any errors remains my own. This article was written at the University of Western Ontario while on sabbatical leave from the Flinders University of South Australia.

2 This assumption is not crucial to the analysis and avoids much notational complexity.

${ }^{3}$ This paper considers only the cases of no recall and complete recall. Sequential search problems with uncertain recall have been analyzed by Karni and Schwartz [3] and Landsberger and Peled [4]

${ }^{4}$ The proof of this statement is given in [6, pp. 14-15]. ${ }^{5}$ In [6, pp. 16-21] it is proved that the following set of conditions is sufficient for the optimal search strategy to be sequential: $\delta=0$; $J=\infty$; no financial search costs; psychic costs $K(\cdot)$ are identical in each period, non-negative and convex; the searcher has complete recall; and observations are i.i.d.

${ }^{6}$ In [2, pp. 605-606] Gal, et al. show $\mathrm{y}^{*}$ exists and is constant across decision points. 


\section{References}

[1] DeGroot, M., Optimal Statistical Decisions, McGraw-Hill, New York, 1970.

[2] Gal, S., Landsberger, M. and B. Levykson, "A Compound Strategy for Search in the Labor Market," International Economic Review 22 (1981), 597-608.

[3] Karni, E. and A. Schwartz, "Search Theory: The Case of Search with Uncertain Recal1," Journal of Economic Theory 16 (1977), 38-52.

[4] Landsberger, M. and D. Peled, "Duration of Offers, Price Structure and the Gain from Search," Journal of Economic Theory 16 (1977), 17-37.

[5] Manning, R. and P. Morgan, "Search and Consumer Theory," forthcoming, Review of Economic Studies (1982).

[6] Manning, R. and P. Morgan, "Optimal Search," mimeographed at the Flinders University of South Australia, 1981.

[7] Rothschild, M., "Searching for the Lowest Price when the Distribution of Prices is Unknown," Journal of Political Economy 82 (1974), 689-711.

[8] Stigler, G., "The Economics of Information," Journal of Political Economy 69 (1961), 213-225.

[9] Stigler, G., "Information in the Labour Market," Journal of Political Economy 70, supplement (1962), 94-105. 